Series A

I. MATHEMATICA

398

\title{
ÜBER EINE DETERMINANTENIDENTITÄT UND DEN ERSTEN FAKTOR DER KLASSENZAHL DES KREISKÖRPERS
}

VON

SEPPO HYYRÖ

HELSIN K I 1967

S U O M A L A N E N T IEDEAKATEMIA

doi:10.5186/aasfm.1967.398 
Am 13. Januar 1967 vorgelegt von P. J. Myrberg und K. Inkeri 


\section{Über eine Determinantenidentität und den ersten Faktor der Klassenzahl des Kreiskörpers}

Wir betrachten für ein gegebenes Polynom $F$ das Produkt

$$
\prod F\left(x_{1}, \ldots, x_{m}\right),
$$

wobei $x_{i}$ für $i=1, \ldots, m$ die Zahlen $\alpha_{i j}(j=1, \ldots, n(i))$ durchläuft. Das Hauptziel des vorliegenden Aufsatzes ist, über eine Methode zu diskutieren für Darstellung von (1) als eine $n(1) \ldots n(m)$-reihige Determinante mit Elementen aus dem Ring der Koeffizienten von $F$ und der symmetrischen Grundfunktionen der $n(i)$ Zahlen $\alpha_{i 1}, \ldots, \alpha_{i n(i)}(i=1, \ldots, m)$. Dies kommt z.B. in der Theorie der Klassenzahl des Kreiskörpers zur Anwendung (s. Nr. 2).

1. Determinantenidentität. Es sei $F\left(x_{1}, \ldots, x_{m}\right)$ ein gegebenes Polynom. Es seien $\alpha_{i 1}, \ldots, \alpha_{i n(i)}(i=1, \ldots, m)$ gegebene Zahlen, wobei $n(1), \ldots, n(m)$ natürliche Zahlen sind. Wir setzen

$$
f_{i}(x)=x^{n(i)}-\left(x-\alpha_{i 1}\right) \ldots\left(x-\alpha_{i n(i)}\right) \quad(i=1, \ldots, m) .
$$

Dann sind die Koeffizienten von $f_{i}(x)$, von den Vorzeichen abgesehen, symmetrische Grundfunktionen der Zahlen $\alpha_{i 1}, \ldots, \alpha_{i n(i)}$. Gibt es in $F\left(x_{1}, \ldots, x_{m}\right)$ Potenzen von $x_{i}$, deren Exponenten $\geqq n(i)$ sind, so wenden wir, nötigenfalls wiederholt, die Substitution

$$
x_{i}^{n(i)}=f_{i}\left(x_{i}\right) \quad(i=1, \ldots, m)
$$

an, so dass das Polynom $F\left(x_{1}, \ldots, x_{m}\right)$ die Form $F_{1}\left(x_{1}, \ldots, x_{m}\right)$ bekommt, wobei der Grad von $F_{1}$ in bezug auf $x_{i}$ höchstens gleich $n(i)-1$ ist. Dabei bleibt das Produkt (1) unverändert.

Für $i=1, \ldots, m ; j=0, \ldots, N-1, \quad$ mit $N=n(1) \ldots n(m)$, definieren wir die Zahlen $r(i, j)$, indem wir $q_{0}=j$ und dann der Reihe nach für $i=1, \ldots, m$

$$
q_{i-1}=q_{i} n(i)+r(i, j), \quad 0 \leqq r(i, j)<n(i)
$$

mit ganzen $q_{1}, \ldots, q_{m}$ setzen. Weiter setzen wir 


$$
\begin{aligned}
& y_{i}=x_{1}^{r(1, i)} \ldots x_{m}^{r(m, i)} \quad(i=0, \ldots, N-1) \\
& Y=\left(y_{0}, \ldots, y_{N-1}\right) .
\end{aligned}
$$

Nun können wir kurz

$$
F_{1}\left(x_{1}, \ldots, x_{m}\right)=c_{00} y_{0}+\ldots+c_{0, N-1} y_{N-1}=G(Y)
$$

schreiben. Die Zahlen $c_{i j}$ (für $i>0$ ) sind durch die Gleichungen

$$
y_{i} G(Y)=\sum_{j=0}^{N-1} c_{i j} y_{j} \quad(i=0, \ldots, N-1)
$$

definiert, wobei die Potenzen von $x_{i}$ mit den Exponenten $\geqq n(i)$ $(i=1, \ldots, m)$ mittels (2) eliminiert sind.

Wir haben den

Satz. Das Produkt (1) ist gleich der Determinante

$$
\left|c_{i j}\right| \quad(i, j=0, \ldots, N-1) .
$$

Für den Beweis führen wir noch die Bezeichnungen

$$
\begin{array}{r}
s(i, j)=r(i, j)+1 \quad(i=1, \ldots, m ; j=0, \ldots, N-1), \\
a_{i j}=\alpha_{1 s(1, j)}^{r(1, i)} \ldots \alpha_{m s(m, j)}^{r(m, i)} \quad(i, j=0, \ldots, N-1), \\
A_{i}=\left(a_{0 i}, \ldots, a_{N-1, i}\right) \quad(i=0, \ldots, N-1)
\end{array}
$$

ein. Dabei ist

$$
F\left(\alpha_{\left.1 s_{1} 1, i\right)}, \ldots, \alpha_{m s(m, i)}\right)=G\left(A_{i}\right) \quad(i=0, \ldots, N-1) .
$$

Im Beweis brauchen wir den Hilfssatz:

Die verallgemeinerte Vandermondesche Determinante

$$
\left|a_{i j}\right| \quad(i, j=0, \ldots, N-1)
$$

kann mittels der Vandermondeschen Determinanten

$$
\left|\alpha_{k j}^{i}\right| \quad(i=0, \ldots, n(k)-1 ; j=1, \ldots, n(k))
$$

in der Form

$$
\left|\alpha_{1 j}^{i}\right|^{N / n(1)} \ldots\left|\alpha_{m j}^{i}\right|^{N / n(m)}
$$

dargestellt werden.

Die Matrix $\left(a_{i j}\right)$ ist nämlich von der Form $B_{1} B_{2}$ mit

$$
\begin{gathered}
B_{1}=\left(\delta_{i j} B\right) \quad(i, j=1, \ldots, n(m)), \\
B_{2}=\left(\alpha_{m j}^{i} I\right) \quad(i=0, \ldots, n(m)-1 ; j=1, \ldots, n(m)),
\end{gathered}
$$


wobei $\delta_{i j}$ das Kroneckersche Symbol, $B$ eine $q \times q$-Matrix $(q=N / n(m))$ und $I$ die $q$-reihige Einheitsmatrix bedeutet. Daher ist

$$
\left|a_{i j}\right|=|B|^{n(m)}\left|\alpha_{m j}^{i}\right|^{q},
$$

woraus der Hilfssatz durch Induktion folgt.

Für den Beweis des Satzes setzen wir zuerst voraus, dass für jedes $i$ die Zahlen $\alpha_{i 1}, \ldots, \alpha_{i n(i)}$ verschieden sind. Dann ist jede Determinante in (5), damit auch die Determinante $\left|a_{i j}\right|$ von Null verschieden. In diesem Fall folgt der Satz also aus

$$
\left|c_{i j}\right|\left|a_{i j}\right|=\left|a_{i j} G\left(A_{j}\right)\right|=G\left(A_{0}\right) \ldots G\left(A_{N-1}\right)\left|a_{i j}\right| .
$$

Sind nötigenfalls einige der Zahlen $\alpha_{i j}$ durch Veränderliche ersetzt, so gilt der Satz nach dem Obigen in einer »Umgebung» der Werte $\alpha_{i j}$. Aus der Stetigkeit der Funktionen (1) und (3) ergibt sich nun, dass der Satz auch für die $\alpha_{i j}$ richtig ist.

2. Anwendungen. Als ein Beispiel sei ein Ausdruck für den ersten Faktor der Klassenzahl des Kreiskörpers der $p^{u}$-ten Einheitswurzeln diskutiert, wobei $p$ eine ungerade Primzahl und $u$ eine natürliche Zahl $\geqq 2$ bedeutet. Es sei $g$ eine primitive Wurzel $\left(\bmod p^{u}\right)$ und $g_{i}$ der kleinste positive Rest von $g^{i}\left(\bmod p^{u}\right)$. Weiter sei

$$
\begin{gathered}
H(x)=g_{0}+g_{1} x+\ldots+g_{t} x^{t}, \quad t=\varphi\left(p^{u}\right)-1 \\
w=\varphi\left(p^{u-1}\right) / 2, \quad v=(p-1) w
\end{gathered}
$$

gesetzt. Dann lässt sich der genannte erste Faktor in der Form

$$
h_{1}=2^{-v} p^{-v u+1} h_{1}^{\prime} \prod H(\Theta)
$$

darstellen, wobei $h_{1}^{\prime}$ den ersten Faktor der Klassenzahl des Kreiskörpers der $p^{u-1}$-ten Einheitswurzeln bezeichnet und $\Theta$ die Wurzeln der Gleichung

$$
\left(x^{p w}+1\right) /\left(x^{w}+1\right)=0
$$

durchläuft (s. [1]). Unter Anwendung von (8) kann man

$$
\Theta^{i} H(\Theta)=\sum_{j=0}^{v-1} b_{i j} \Theta^{j} \quad(i=0, \ldots, v-1)
$$

schreiben. Also ist das Produkt $\Pi H(\Theta)$ in (7) gleich der Determinante $\left|b_{i j}\right|$. Dies ist ein Ergebnis von Lepistö [1], Nr. 35 (abgesehen vom Vorzeichen und von der Reihenfolge der Zeilen der Determinante). Die Begründungsweise (6) (für $m=1$ ) ist jedoch einfacher als die von [1]. 
T. Metsänkylä hat dem Verfasser mitgeteilt, dass er Betrachtungen von [1] in einem allgemeinen Kreiskörper generalisiert hat, wobei Determinantendarstellung für Produkte der Form (1) mit $m>1$ zur Anwendung kommt. Hier sei nur auf die Arbeit [2] hingewiesen.

3. Über eine Abschätzung von LePIsTö [1]. Es sei hierbei noch bemerkt, dass die erste Abschätzung (8.10) in [1] sich in die Form $K \leqq 2^{1+(u-5) w / 2}$ verbessern lässt, wenn die Formeln (10.5) und (8.4) statt (8.7) angewendet werden. Dadurch ergibt sich für den ersten Faktor der Klassenzahl des Kreiskörpers der $2^{u}$-ten Einheitswurzeln $(u \geqq 3)$ statt der Abschätzung (8.11) von [1] die etwas bessere Abschätzung

$$
h_{1}\left(2^{u}\right) \leqq 2^{U}, \quad U=(u-6) 2^{u-3}+u .
$$

Universität Oulu, Finnland

und

Universität Turku, Finnland 


\section{Literatur}

[1] Lepistö, T.: On the first factor of the class number of the cyclotomic field and Dirichlet's $L$-functions. - Ann. Acad. Sci. Fenn. A I, 387 (1966), 1-53.

[2] Metsänkylä, T.: Über den ersten Faktor der Klassenzahl des Kreiskörpers (in Vorbereitung). 